\title{
Nonlinear thermal instability in a horizontal porous layer with an internal heat source and mass flow
}

Received: date / Accepted: date

\begin{abstract}
Linear and nonlinear stability analyses of Hadley-Prats flow in a horizontal fluid-saturated porous medium with a heat source are studied. The results indicate that, in the linear case, an increase in the horizontal thermal Rayleigh number is stabilizing for both positive and negative values of mass flow. In the nonlinear case, a destabilizing effect is identified at higher mass flow rates. An increase in the heat source has a destabilizing effect. Qualitative changes appear in $R_{z}$ as the mass flow moves from negative to positive for different internal heat sources.
\end{abstract}

Keywords Nonlinear stability analysis · Heat source · Porous medium · Mass flow

\section{Introduction}

Thermal convection driven by an internal heat source with a horizontal mass flow has many practical applications such as underground energy transport, cooling of nuclear reactors, food processing, oil recovery, underground storage of waste products and thermal convection in clouds [1-4].

In the last few decades, convection involving internal heat sources has attracted particular research attention. Early experimental investigations include Schwiderski et al. [5] and Tritton et al. [6], with Roberts [7] and Thirlby [8] providing theoretical analyses. Parthiban and Patil [9] investigated thermal convection due to non-uniform heating boundaries with inclined thermal gradients in the presence of an internal heat source, followed by an extension to anisotropic porous layers by Parthiban and Patil [10]. The case of an inclined layer with internal heat source was analyzed by Barletta et al. [11], where both boundaries were isothermal and kept at the same temperatures. Rionero and Straughan [12] investigated the linear and nonlinear effects in the presence of variable gravity effect and heat generation. Extensive reviews of the theory and applications can be found in the article by Alex and Patil [13]. Hill [14] investigated a porous layer with concentration based internal heat generation, with linear and nonlinear stability analyses of thermosolutal convection. Chamka [15] analyzed the effect of an internal heat source or sink for hydromagnetic simultaneous heat and mass transfer by utilising similarity solutions. Thermosolutal convection in a saturated anisotropic porous medium with internal heat generation is reported by Bhadauria [16]. Borujerdi et. al [17] examined the steady state heat conduction with a uniform heat source where the solid and fluid phase are at different temperatures. Borujerdi et. al [18] study the influence of Darcy number on the critical Rayleigh number in onset of

A. Matta P.A.L. Narayana

Department of Mathematics, Indian Institute of Technology Hyderabad, Hyderabad - 502205, Telangana, India

E-mail: ananth@iitm.ac.in

A. A. Hill

Department of Biological, Biomedical and Analytical Sciences,

University of the West of England, Bristol BS16 1QY, UK

E-mail: antony.hill@uwe.ac.uk 
convection with uniform internal heating. A collection of comprehensive theories and experiments of thermal convection in porous media (with their practical applications), has been surveyed by Nield and Bejan [19]. Capone and Rionero [20] have studied the nonlinear stability analysis of a convective motion in a horizontal porous layer which is driven by a temperature gradient. Several problems on nonlinear stability analyses using the energy method are discussed by Kaloni and his contributors [2125]. In the Lyapunov sense, when the disturbance of the basic flow is unstable linearized theory provide sufficient conditions, whereas nonlinear theory provides sufficient conditions for the disturbance to be asymptotically stable.

The aim of this article is to study the influences of both a heat source and a mass flow. The corresponding eigenvalue problems are solved numerically utilising the shooting and Runga-Kutta method.

\section{Mathematical analysis}

An infinite shallow horizontal fluid saturated porous medium with thickness $d$ is considered. The $z^{\prime}$ -axis is vertically upwards and there is a net flow along the direction of $x^{\prime}$ - axis with magnitude $M^{\prime}$. The vertical temperature difference across the boundaries is $\triangle \theta$. Further imposed is the horizontal temperature gradient vector $\left(\beta_{\theta_{x}}, \beta_{\theta_{y}}\right)$. The porous layer flow is governed by the Darcy law, where the linear Boussinesq approximation is assumed. Utilising the equation of the conservation of energy, the governing equations in dimensional form are

$$
\begin{gathered}
\nabla^{\prime} \cdot q^{\prime}=0, \\
\frac{\mu}{K} q^{\prime}+\nabla^{\prime} P^{\prime}-\rho_{0}\left[1-\gamma_{\theta}\left(\theta^{\prime}-\theta_{0}\right)\right] g=0, \\
(\rho c)_{m}\left(\frac{\partial \theta^{\prime}}{\partial t^{\prime}}\right)+\left(\rho c_{p}\right)_{f} q^{\prime} \cdot \nabla^{\prime} \theta^{\prime}=k_{m} \nabla^{\prime 2} \theta^{\prime}+Q^{\prime},
\end{gathered}
$$

with the following boundary conditions:

$$
w^{\prime}=0, \quad \theta^{\prime}=\theta_{0}-\frac{1}{2}( \pm \triangle \theta)-\beta_{\theta_{x}} x^{\prime}-\beta_{\theta_{y}} y^{\prime} \quad \text { at } \quad z^{\prime}= \pm \frac{d}{2} .
$$

Here, the Darcy velocity is defined as $q^{\prime}=\left(u^{\prime}, v^{\prime}, w^{\prime}\right), P^{\prime}$ is the pressure, $\theta^{\prime}$ is temperature and $Q^{\prime}$ is an internal heat source. The subscripts $f$ and $m$ are refer to the fluid and the porous medium, respectively. $\phi$ and $K$ are the porosity and permeability of the porous layer. $c, \rho, \mu, k_{m}$ and $\gamma_{\theta}$ denote the specific heat, density, viscosity, thermal diffusivity, and thermal expansion coefficient in the porous medium, respectively.

The following dimensionless variables are introduced to non-dimensionalize the governing equations:

$$
\begin{gathered}
(x, y, z)=\frac{1}{d}\left(x^{\prime}, y^{\prime}, z^{\prime}\right), \quad t=\frac{\alpha_{m} t^{\prime}}{a d^{2}}, \quad q=\frac{d q^{\prime}}{\alpha_{m}}, \quad P=\frac{K\left(P^{\prime}+\rho_{0} g z^{\prime}\right)}{\mu \alpha_{m}}, \\
\theta=\frac{R_{z}\left(\theta^{\prime}-\theta_{0}\right)}{\triangle \theta}, \quad M=\frac{d M^{\prime}}{\alpha_{m}}, \quad Q=\frac{d^{2} Q^{\prime}}{k_{m} \triangle \theta}
\end{gathered}
$$

where

$$
\alpha_{m}=\frac{k_{m}}{\left(\rho c_{p}\right)_{f}}, \quad a=\frac{(\rho c)_{m}}{\left(\rho c_{p}\right)_{f}}, \quad R_{z}=\frac{\rho_{0} g \gamma_{\theta} K d \triangle \theta}{\mu \alpha_{m}} .
$$

Here, $R_{z}$ denote the vertical thermal Rayleigh number. The horizontal thermal Rayleigh numbers are defined as follows

$$
R_{x}=\frac{\rho_{0} g \gamma_{\theta} K d^{2} \beta_{\theta_{x}}}{\mu \alpha_{m}}, \quad R_{y}=\frac{\rho_{0} g \gamma_{\theta} K d^{2} \beta_{\theta_{y}}}{\mu \alpha_{m}} .
$$


The previous scaling for dimensional variables and the horizontal thermal Rayleigh numbers was introduced by Weber [27] and used extensively by Nield [28]. Under these dimensionless variables, the governing Eqs. (1) - (3) are

$$
\begin{gathered}
\nabla \cdot q=0, \\
q+\nabla P-\theta \mathbf{k}=0, \\
\frac{\partial \theta}{\partial t}+q \cdot \nabla \theta=\nabla^{2} \theta+Q R_{z},
\end{gathered}
$$

with the conditions of the plates being

$$
w=0, \quad \theta=-\frac{1}{2}\left( \pm R_{z}\right)-R_{x} x-R_{y} y \quad \text { at } \quad z= \pm \frac{1}{2}
$$

From Eqs. (8) - (10) we observe that all of the thermal Rayleigh numbers are involved in boundary conditions (11). The condition on temperature at both bounding planes give a linear variation of temperature. This spatial linear variation on temperature along the horizontal planes bounding a fluid layer is a physically more realistic situation than the strictly uniform heating Capone and Rionero [20]. However, in the present problem uniform heating can be recovered by setting the horizontal thermal gradients to zero.

\section{Steady-state Solution}

The flow governing equations (8)-(10), subject to (11), has a basic state solution of the form

$$
\begin{gathered}
\theta_{s}=\tilde{\theta}(z)-R_{x} x-R_{y} y, \\
u_{s}=u(z), \quad v_{s}=v(z), \quad w_{s}=0, \quad P_{s}=P(x, y, z),
\end{gathered}
$$

with

$$
\begin{gathered}
u_{s}=-\frac{\partial P}{\partial x}, \quad v_{s}=-\frac{\partial P}{\partial y} \\
0=-\frac{\partial P}{\partial z}+\widetilde{\theta}(z)-R_{x} x-R_{y} y \\
D^{2} \widetilde{\theta}=-u_{s} R_{x}-v_{s} R_{y}-Q R_{z} .
\end{gathered}
$$

Here $D=\frac{d}{d z}$, and we have a net flow $\mathrm{M}$ in the horizontal direction such that $\int_{-1 / 2}^{1 / 2} u(z) d z=M$ and $\int_{-1 / 2}^{1 / 2} v(z) d z=0$. The solution in the form of flow velocity and temperature in the medium is then given by

$$
\begin{gathered}
u_{s}=R_{x} z+M, \quad v_{s}=R_{y} z \\
\widetilde{\theta}=-R_{z} z-\frac{\lambda}{24}\left(4 z^{3}-z\right)-\left(M R_{x}+Q R_{z}\right)\left(\frac{z^{2}}{2}-\frac{1}{8}\right),
\end{gathered}
$$

where $\lambda=R_{x}^{2}+R_{y}^{2}$. 


\section{Perturbation Equations}

We consider the perturbations in the form $q=q_{s}+\bar{q}, \theta=\theta_{s}+\bar{\theta}$ and $P=P_{s}+\bar{P}$. By substituting these perturbations in the dimensionless governing equations (8) - (10), we get

$$
\begin{gathered}
\nabla \cdot \bar{q}=0 \\
\bar{q}=-\nabla \bar{P}+\bar{\theta} \mathbf{k}, \\
\frac{\partial \bar{\theta}}{\partial t}+q_{s} \cdot \nabla \bar{\theta}+\bar{q} \cdot \nabla \theta_{s}+\bar{q} \cdot \nabla \bar{\theta}=\nabla^{2} \bar{\theta}
\end{gathered}
$$

where

$$
\begin{gathered}
\nabla \theta_{s}=-\left(R_{x}, R_{y}, R_{z}-\widetilde{A}\right) . \\
\widetilde{A}=\frac{\lambda}{24}\left[1-12 z^{2}\right]-\left(M R_{x}+Q R_{z}\right) z,
\end{gathered}
$$

The conditions at the plates are

$$
\bar{w}=0, \quad \bar{\theta}=0 \quad \text { at } \quad z= \pm \frac{1}{2}
$$

Note that (19) shows that there are no normal velocity and temperature perturbations at the plates.

\section{Linear Stability Analysis}

To perform a linear stability analysis we neglect the nonlinear terms from Eq. (18). The linearized perturbations equations are then

$$
\begin{gathered}
\nabla \cdot \bar{q}=0, \\
\bar{q}=-\nabla \bar{P}+\bar{\theta} \mathbf{k}, \\
\frac{\partial \bar{\theta}}{\partial t}+q_{s} \cdot \nabla \bar{\theta}+\bar{q} \cdot \nabla \theta_{s}=\nabla^{2} \bar{\theta},
\end{gathered}
$$

where

$$
\nabla \theta_{s}=-\left(R_{x}, R_{y}, R_{z}-\frac{\lambda}{24}\left[1-12 z^{2}\right]+\left(M R_{x}+Q R_{z}\right) z\right) .
$$

The conditions at the plates are

$$
\bar{w}=0, \quad \bar{\theta}=0 \quad \text { at } \quad z= \pm \frac{1}{2} .
$$

Adopting a normal mode solution to Eqs. (20) - (22) of the form

$$
[\bar{q}, \bar{\theta}, \bar{P}]=[q(z), \theta(z), P(z)] \exp \{i(k x+l y)+\sigma t\}
$$

and further eliminating $P$, yields

$$
\begin{gathered}
\left(D^{2}-\alpha^{2}\right) w+\alpha^{2} \theta=0 \\
\left(D^{2}-\alpha^{2}-\left(\sigma+i\left(k u_{s}+l v_{s}\right)\right)\right) \theta+\frac{i}{\alpha^{2}}\left(k R_{x}+l R_{y}\right) D w-(D \widetilde{\theta}) w=0 .
\end{gathered}
$$

Eqs. (25) - (26), subject to $w=\theta=0$ at both the plates $z=\frac{1}{2}$ and $z=-\frac{1}{2}$, constitute an eigenvalue problem for vertical thermal Rayleigh number $R_{z}$ with $a, R_{x}, R_{y}, k$ and $l$ as parameters. In the above, $\alpha=\sqrt{k^{2}+l^{2}}$ is the overall wave number. Numerical results are presented in Section 7 . 


\section{Nonlinear Stability Analysis}

In this section our nonlinear analysis via energy functional is presented as follows. We multiply equations (17) and (18) by $\bar{q}$ and $\bar{\theta}$, respectively, and integrate over $\Omega$, where $\Omega$ denotes a typical periodicity cell. This yields the following identities

$$
\begin{gathered}
\|\bar{q}\|^{2}=\langle\bar{\theta} \bar{w}\rangle, \\
\frac{1}{2} \frac{d\|\bar{\theta}\|^{2}}{d t}=-\left\langle\left(\bar{q} \cdot \nabla \theta_{s}\right) \bar{\theta}\right\rangle-\|\nabla \bar{\theta}\|^{2} .
\end{gathered}
$$

Here $\|\cdot\|$ and $\langle\cdot\rangle$ denote the norm and inner product on $L^{2}(\Omega)$. We adopt the energy functional (cf. [29])

$$
E(t)=\frac{\xi}{2}\|\bar{\theta}\|^{2}
$$

with coupling parameter $\xi>0$. The system of equations (27)-(28) along with Eq. (29), can now be represented in the form

$$
\frac{d E}{d t}=I-\Delta,
$$

where

$$
\begin{gathered}
I=-\xi\left\langle\left(\bar{q} \cdot \nabla \theta_{s}\right) \bar{\theta}\right\rangle+\langle\bar{\theta} \bar{w}\rangle, \\
\Delta=\xi\|\nabla \bar{\theta}\|^{2}+\|\bar{q}\|^{2} .
\end{gathered}
$$

We define

$$
n=\max _{H} \frac{I}{\Delta}
$$

where $H$ is the space of all admissible solutions to equations (16) - (18). If $0<n<1$ it follows that

$$
\frac{d E}{d t} \leq-\Delta(1-n) .
$$

The classical Poincare inequality $\left\|\bar{q}-\bar{q}_{\Omega}\right\|_{L^{p}(\Omega)} \leq C\|\nabla \bar{q}\|_{L^{p}(\Omega)}$, where $\Omega$ is a open connected locally compact Hausdorff space and use of $\bar{q}_{\Omega}=\frac{1}{|\Omega|} \int_{\Omega} \bar{q}(y) d y$ yields

$$
\frac{d E}{d t} \leq-2 \pi^{2}(1-n) \min \left\{1, \frac{a}{L_{e} \phi}\right\} E .
$$

Eq. (35) then guarantees that $E(t) \rightarrow 0$ as $t \rightarrow \infty$ for $0<n<1$. Applying the arithmetic-geometric mean inequality on Eq. (27) yields

$$
\|\bar{q}\|^{2} \leq\|\bar{\theta}\|^{2} .
$$

From Eqs. (36) and (29) it follows that the decay of $\|\bar{q}\|$ is implied by the decay of $E(t)$ and hence the system is stable. From the above, we have identified that the non-linear stability requires the critical argument at $n=1$. The corresponding Euler-Lagrange system with the maximum problem Eq. (33) is

$$
\begin{gathered}
\xi \bar{\theta} \nabla \theta_{s}-\bar{\theta} \mathbf{k}+2 \bar{q}=\nabla \bar{\delta}, \\
\bar{w}-\xi \bar{q} \cdot \nabla \theta_{s}+2 \xi \nabla^{2} \bar{\theta}=0 .
\end{gathered}
$$

Here $\bar{\delta}$ is Lagrange multiplier introduced because $\bar{q}$ is divergence free. We consider $R_{z}$ as the eigenvalue and estimate the maximum variation of $R_{z}$ with optimal choice of $\xi$. Eqs. (37) - (38) yield

$$
\frac{\partial R_{z}}{\partial \xi}=\frac{n\left(1-\xi R_{z}\right)\|\nabla \bar{\theta}\| \|^{2}+\langle\widetilde{A} \bar{\theta} \bar{w}\rangle-R_{x}\langle\bar{\theta} \bar{u}\rangle+R_{y}\langle\bar{\theta} \bar{v}\rangle}{\xi^{2}\left(2 n|| \nabla \bar{\theta} \|^{2}+\langle\widetilde{A} \bar{\theta} \bar{w}\rangle-R_{x}\langle\bar{\theta} \bar{u}\rangle+R_{y}\langle\bar{\theta} \bar{v}\rangle\right)},
$$

Equation (39) is important, and also noted that if $R_{x}=R_{y}=0$ and $Q=0$, we get

$$
\frac{\partial R_{z}}{\partial \xi}=\frac{\left(1-\xi R_{z}\right)}{2 \xi^{2}} .
$$


Equation (40) is same as the expressions reported by Guo and Kaloni [21]. We solve the system of Eqs. (37) - (38) in presence of critical value $n=1$. To evaluate this system numerically, we apply curlcurl of Eq. (37) and further use the third component of the resulting equation

$$
\xi R_{x} \frac{\partial^{2} \bar{\theta}}{\partial x \partial z}+\xi R_{y} \frac{\partial^{2} \bar{\theta}}{\partial y \partial z}+\xi \nabla_{1}^{2}\left[\left(-R_{z}+\widetilde{A}\right) \bar{\theta}\right]+2 \nabla_{1}^{2} \bar{w}-\nabla_{1}^{2} \bar{\theta}-2\left(\frac{\partial^{2} \bar{u}}{\partial x \partial z}+\frac{\partial^{2} \bar{v}}{\partial y \partial z}\right)=0,
$$

where $\nabla_{1}^{2}=\left(\frac{\partial^{2}}{\partial x^{2}}+\frac{\partial^{2}}{\partial y^{2}}\right)$. Now, we apply the normal mode expansion

$$
[\bar{q}, \bar{\theta}, \bar{\delta}]=[q(z), \theta(z), \delta(z)] \exp (i(k x+l y))
$$

with $\left(R_{x}, R_{y}\right) \cdot(k, l)=0$, (Nield [26] and Kaloni and Qiao [22]), i.e the horizontal thermal Rayleigh number vector is orthogonal to wave number vector. We substitute (42) in Eqs. (37), (38), (41) and eliminate $u, v$ and $\delta$ to obtain

$$
\begin{gathered}
D^{2} w=\frac{\alpha^{2}}{2}\left(2 w+\xi\left[-R_{z}+\widetilde{A}\right] \theta-\theta\right) \\
D^{2} \theta=\frac{1}{2}\left[-R_{z}+\widetilde{A}-\xi^{-1}\right] w+\left[\alpha^{2}-\xi\left(\frac{R_{x}^{2}+R_{y}^{2}}{4}\right)\right] \theta .
\end{gathered}
$$

The system of Eqs. (43) to (44) is evaluated with the boundary conditions $w=\theta=0$ at $z= \pm \frac{1}{2}$. The critical vertical thermal Rayleigh number is obtained as $R_{z}=\max _{\xi} \min _{\alpha^{2}} R_{z}$.

\section{Results and Discussion}

The onset of thermal convection in a fluid-saturated porous layer in the presence of mass flow and an internal heat source effect is analyzed using both linear and nonlinear stability theory. Both cases are studied based on the classical normal mode technique. We treat the vertical thermal Rayleigh number as the eigenvalue $R_{z}$. Here, the critical vertical thermal Rayleigh number $R_{z}$ is defined as the minimum of all $R_{z}$ values as the wave number $\alpha$ is varied. The vector of wave number is defined as $\alpha=(k, l, 0)$. To achieve the stationary convection boundary, we set $\sigma=0$ (the removal of the oscillatory mode is discussed in the Appendix), with $\left(R_{x}, R_{y}\right) \cdot(k, l)=0$. The term longitudinal disturbances are characterized by $k=0$. In the same way, transverse disturbances are characterized by $l=0$. In Table $1, R_{z_{l}}$ and $R_{z_{e}}$ indicate the linear and nonlinear critical thermal Rayleigh number $\left(R_{z}\right)$. $\alpha_{l}$ and $\alpha_{e}$ indicate the critical wave number in linear and nonlinear cases. From Table 1, it is observed that when

Table 1 Critical thermal Rayleigh numbers at $M=0$.

\begin{tabular}{|c|c|ccccc|}
\hline & $R_{x}$ & 0 & 10 & 20 & 30 & 40 \\
\hline \multirow{3}{*}{$Q=0$} & & & & & & \\
& $R_{z_{l}}$ & 39.4784 & 42.0076 & 49.5486 & 61.9566 & 78.9663 \\
& $\alpha_{l}$ & 3.13999 & 3.1399 & 3.1499 & 3.1599 & 3.2199 \\
& $R_{z_{e}}$ & 39.47840 & 40.72345 & 44.20928 & 49.18550 & 53.61943 \\
& $\alpha_{e}$ & 3.13999 & 3.08999 & 2.94999 & 2.7199 & 2.2799 \\
& $R_{z_{l}}$ & 39.2360 & 41.7294 & 49.1460 & 61.2757 & 77.7028 \\
& $\alpha_{l}$ & 3.1599 & 3.1599 & 3.1699 & 3.2099 & 3.3099 \\
& $R_{z_{e}}$ & 39.05626 & 40.27496 & 43.67935 & 48.51113 & 52.6681 \\
& $\alpha_{e}$ & 3.15999 & 3.109999 & 2.96999 & 2.73999 & 2.28999 \\
& $R_{z_{l}}$ & 38.53950 & 40.93195 & 47.99451 & 59.34272 & 74.11823 \\
& $\alpha_{l}$ & 3.19999 & 3.19999 & 3.22999 & 3.33999 & 3.59999 \\
& $R_{z_{e}}$ & 37.85142 & 38.99556 & 42.16919 & 46.59001 & 49.8985 \\
& $\alpha_{e}$ & 3.20999 & 3.15999 & 3.0299 & 2.7999 & 2.27999 \\
\hline
\end{tabular}

$Q=0$ and $M=0$, in the linear case, the results are in very good agreement with earlier published results in the literature, Nield [26]. For an increase in the value of $Q$ from 0 to 2, the critical value of $R_{z}$ is reduced seen in Table 1 in both cases. Hence, the heat flow parameter causes destabilization in the medium. A fixed notation is used to represent the curves corresponding to the linear and nonlinear results. Dotted lines represent linear stability results and solid lines represents nonlinear stability results in Figs. 1 to 3. 


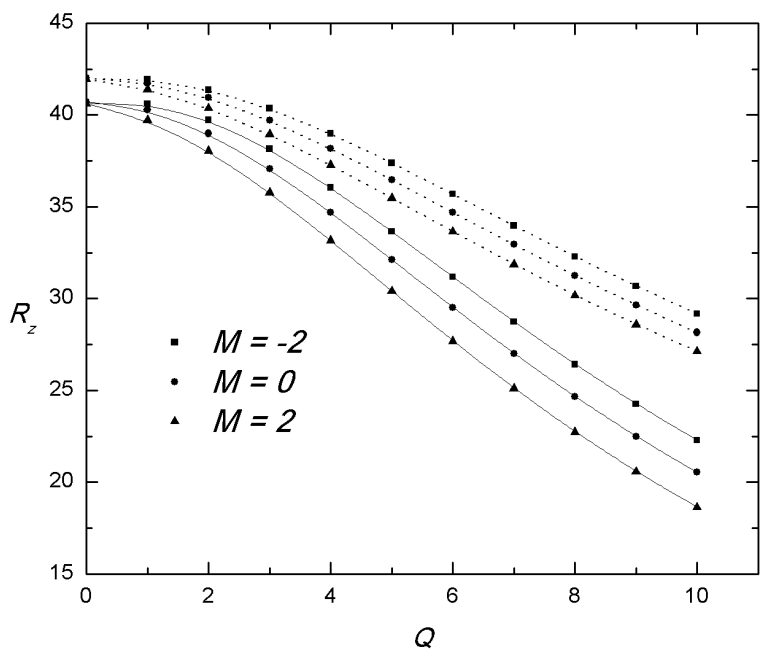

Fig. 1 Variation of $R_{z}$ with $Q$ at $R_{x}=10$ and $R_{y}=0$.

A comparison of the critical value of $R_{z}$ as a function of $Q$ for different values of the mass flow rate $M$ is shown in Fig. 1 at $R_{x}=10$ and $R_{y}=0$. It is observed that, as $Q$ increases the critical values of $R_{z}$ decreases. However, as $M$ increases from -2 to 2 , at a higher value of $M$, the critical $R_{z}$ value is lower than at lower values of $M$ in both the cases as seen in Fig. 1. For both positive and negative values of $M, R_{z}$ decreases with increasing values of $Q$. It indicates that increasing the heat source has a strongly destabilizing effect. This is due to the fact that the global temperature of the system is increasing with increasing heat source and causes the instability. As increasing the heat source the threshold critical region between linear and nonlinear results is increasing as seen in Fig. 1.

The response of $R_{z}$ with varying $R_{x}$ is shown in Fig. 2 for negative and positive values of $M$.

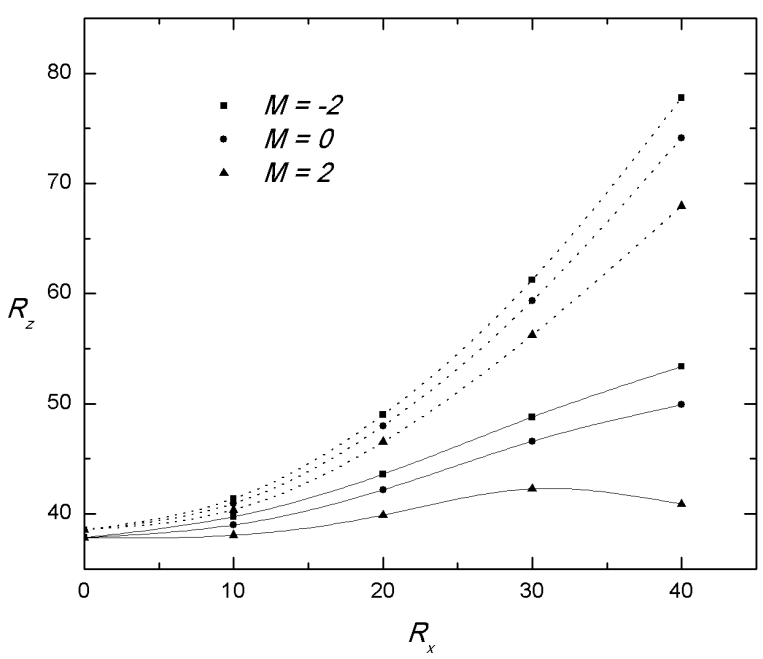

Fig. 2 Variation of $R_{z}$ with $R_{x}$ at $Q=1$ and $R_{y}=0$.

It is noted that, at $M=-2$ in the linear case, the critical value of $R_{z}$ is higher than the remaining all values of $M$. When $R_{x}$ is increased the corresponding $R_{z}$ values also increase for all $M$ values. This indicates that the flow rate is strongly stabilizing in the linear case as compared to nonlinear case seen in Fig. 2. It is also interesting to observe that, as $R_{x}$ increases the critical value of $R_{z}$ also increases up 
to certain value of $R_{x}$ thereafter the $R_{z}$ value decreases for $M=2$ in nonlinear case seen in Fig. 2 . It means that, the flow rate is strongly destabilizing at higher values of $R_{x}$ and $M$ in the nonlinear case.

Fig. 3 shows the response of $R_{z}$ with mass flow rate $M$ in the presence of different values of $Q=0,1,2$ for $R_{x}=10$.

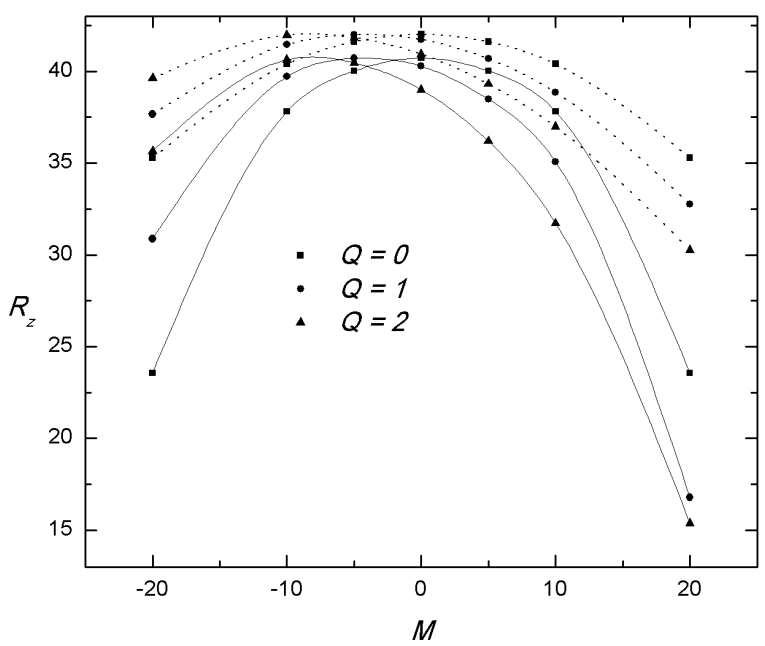

Fig. 3 Variation of $R_{z}$ with $M$ at $R_{x}=10$ and $R_{y}=0$.

As mass flow rate $(M)$ increases $R_{z}$ also increases up to certain values of $M$ thereafter decreases for all values of $Q$ as demonstarted in Fig. 3. $Q$ has a strongly stabilizing effect up to certain value of $M$, then after strongly destabilizes the flow in both cases. It is seen that, increasing the magnitude of horizontal mass flow in both negative and positive directions, the critical value of $R_{z}$ is decreased.

\section{Conclusion}

We have analyzed the instability of thermal convection in Hadley-Prats flow subject to an internal heat source using linear and nonlinear stability analysis. The results yield the following conclusions:

- An increase in the internal heat source causes a strong destabilization in all cases, as it raises the global temperature of the system.

- In the presence of horizontal mass flow, the flow is stabilizing at higher horizontal Rayleigh numbers in the linear case whereas it is destabilizing in the nonlinear case at larger mass flows.

- Qualitative changes appear in $R_{z}$ as the mass flow moves from negative to positive for different internal heat sources.

Acknowledgment: The authors are grateful to the anonymous referees for remarks which improved the work considerably.

\section{Appendix}

In this section we show that the imaginary part of $\sigma$ is 0 (i.e. the oscillatory mode does not exist). After we use the condition $\left(R_{x}, R_{y}\right) \cdot(k, l)=0$, Eqs. $(25)-(26)$ transformed as

$$
\begin{gathered}
\left(D^{2}-\alpha^{2}\right) w+\alpha^{2} \theta=0 \\
\left(D^{2}-\alpha^{2}-\sigma-i M k\right) \theta-(D \widetilde{\theta}) w=0
\end{gathered}
$$


subject to boundary conditions $w=\theta=0$ at both the plates $z=\frac{1}{2}$ and $z=-\frac{1}{2}$. By eliminating $\theta$, for a single equation obtained from Eqs. (45) - (46) yields

$$
\left(D^{2}-\alpha^{2}\right)^{2} w-(\sigma+i M k)\left(D^{2}-\alpha^{2}\right) w+\alpha^{2}(D \tilde{\theta}) w=0 .
$$

Multiplying Eq. (47) by $\bar{w}$ (complex conjugate of $w$ ) and integrating by parts over $\left[-\frac{1}{2}, \frac{1}{2}\right]$, and using the boundary condition we obtain

$$
\left\|D^{2} w\right\|^{2}+2 \alpha^{2}\|D w\|^{2}+\alpha^{4}\|w\|^{2}+(\sigma+i M k)\left(\|D w\|^{2}+\alpha^{2}\|w\|^{2}\right)+\alpha^{2} \int_{-0.5}^{0.5}(D \widetilde{\theta})|w|^{2} d z=0 .
$$

Taking the imaginary part of Eq. (48) when $\sigma=\sigma_{r}+i \sigma_{i}$

$$
\sigma_{i}\left(\|D w\|^{2}+\alpha^{2}\|w\|^{2}\right)=-M k\left(\|D w\|^{2}+\alpha^{2}\|w\|^{2}\right) .
$$

When $k=0$, we have $\sigma_{i}\left(\|D w\|^{2}+\alpha^{2}\|w\|^{2}\right)=0$, implies $\sigma_{i}=0$.

This shows that the stationary longitudinal mode is the only possible mode for the convection induced by horizontal mass flow as stated by Nield and Bejan [19] and Kaloni and Qiao [22].

\section{References}

1. Chen, X., Li, A.: An experimental study on particle deposition above near-wall heat source. Building and Environment 81, 139-149 (2014)

2. Aihara, T., Fu, W., Shimoyama, M.H.T.: Experimental study of heat and mass transfer from a horizontal cylinder in downward air-water mist flow with blockage effect. Experimental thermal and fluid science 3, 623-631 (1990)

3. Bendrichi, G., Shemilt, L.W.: Mass transfer in horizontal flow channels with thermal gradients. The Canadian Journal of Chemical Engineering 75, 1067-1074 (1997)

4. Kwon, O., Bae, K., Park, C.: Cooling characteristics of ground source heat pump with heat exchange methods. Renewable Energy 71, 651-657 (2014)

5. Schwiderskei,. W., Schwabh, J.A.: Convection experiments with electrolytically heated fluid layers. J. Fluid Mech. 48, 703-719 (1971)

6. Trittond, J., Zarraua, M.N.: Convection in horizontal layers with internal heat generation. J. Fluid Mech. 30, 21-31 (1967)

7. Roberts, P.H.: Convection in horizontal layers with internal heat generation: Theory. J. Fluid Mech. 30 , 33-49 (1967)

8. Thirlby, R.: Convection in an internally heated layer. J. Fluid Mech. 44, 673-693 (1970)

9. Parthiban, C., Patil, P.R.: Effect of non-uniform boundary temperatures on thermal instability in a porous medium with internal heat source. Int. Commun. Heat Mass Transfer 22, 683-692 (1995)

10. Parthiban, C., Patil, P.R.: Thermal instability in an anisotropic porous medium with internal heat source and inclined temperature gradient. Int. Comm. Heat Mass Transfer 24, 1049-1058 (1997)

11. Barletta, A., Celli, M., Nield, D.A.: Unstable buoyant flow in an inclined porous layer with an internal heat source. Int. J. Thermal Sciences 79, 176-182 (2014)

12. Rionero, S., Straughan, B.: Convection in a porous medium with internal heat source and variable gravity effects. Int. J. Eng. Sci. 28, 497-503 (1990)

13. Alex, S.M., Patil, P.R.: Effect of a variable gravity field on convection in an anisotropic porous medium with internal heat source and inclined temperature gradient. Transactions of the ASME 144-150 (2002)

14. Hill, A.A.: Double-diffusive convection in a porous medium with a concentration based internal heat source. Proc. R. Soc. A 461, 561-574 (2005)

15. Chamka, A.J., Raheem, A., Khaled, A.: Similarity solutions for hydromagnetic simultaneous heat and mass transfer by natural convection from an inclined plate with internal heat generation or absorption. J. Heat Mass Transfer 37, 117-123 (2001)

16. Bhadauria, B.S.: Double diffusive convection in a saturated anisotropic porous layer with internal heat source. Transp. Porous Media 92, 299-320 (2012)

17. Borujerdi, A., Noghrehabadi, A.R., Rees, D.A.S.: Onset of convection in a horizontal porous channel with uniform heat generation using a thermal nonequilibrium model. Transp. Porous Med. 69, 343-357 (2007)

18. Borujerdi, A.N., Noghrehabadi, A.R., Rees, D.A.S.: Influence of Darcy number on the onset of convection in a porous layerwith a uniform heat source. Int. J. of Thermal Sciences 47, 1020-1025 (2008)

19. Nield, D.A., Bejan, A.: Convection in Porous Media. 4th edn. Springer, Berlin (2013)

20. Capone, F., Rionero, S.: Nonlinear stability of a convective motion in a porous layer driven by a horizontally temperature gradient, Continuum Mech. Thermodynamics 15 529-538 (2003)

21. Guo, J., Kaloni, P.N.: Nonlinear stability and convection induced by inclined thermal and solutal gradients. Z. Angew. Math. Phys. 46, 645-654 (1995) 
22. Kaloni, P.N., Qiao, Z.: Non-linear stability of convection in a porous medium with inclined temperature gradient. Int. J. Heat Mass Transfer 40, 1611-1615 (1997)

23. Kaloni, P.N., Qiao, Z.: Nonlinear convection induced by inclined thermal and solutal gradients with mass flow. Continuum Mech. Thermodyn. 12, 185-194 (2000)

24. Kaloni, P.N., Qiao, Z.: Non-linear convection in a porous medium with inclined temperature gradient and variable gravity effects. Int. J. Heat Mass Transfer 44, 1585-1591 (2001)

25. Kaloni, P.M., Lou, J.X.: Nonlinear convection of a viscoelastic fluid with inclined temperature gradient. Continuum Mech. Thermodyn. 17, 17-27 (2005)

26. Nield, D.A.: Convection in a porous medium with inclined temperature gradient: additional result., Int. J. Heat Mass Transfer 37, 3021-3025 (1994)

27. Weber, J.E.: Convection in a porous medium with horizontal and vertical temperature gradients. Int. J. Heat Mass Transfer 17, 241-248 (1974)

28. Nield, D.A.: Convection in a porous medium with inclined temperature gradient and horizontal mass flow. Heat Transfer 5, 153-185 (1990)

29. Straughan, B.: The Energy Method, Stability, and Nonlinear Convection. Springer, New York (2010) 